\title{
Supporting Information for \\ Mobility of Four Common Mercury Species in Model and Natural Unsaturated Soils
}

\author{
Ke Gai $^{1,2}$, Thomas P. Hoelen ${ }^{3}$, Heileen Hsu-Kim ${ }^{2,4}$, Gregory V. Lowry ${ }^{1,2, *}$
}

${ }^{1}$ Civil \& Environmental Engineering, Carnegie Mellon University, Pittsburgh, Pennsylvania 15213, United States

${ }^{2}$ Center for the Environmental Implications of NanoTechnology (CEINT)

${ }^{3}$ Chevron Energy Technology Company, San Ramon CA 94583, United States

${ }^{4}$ Civil \& Environmental Engineering, Duke University, Durham, North Carolina 27708, United

States

Number of pages: 20

Table of Contents

S1. Tracer test analysis.

S2. $\mathrm{Hg}(\mathrm{II})^{*}$ speciation analysis.

Table S1. Column experiment parameters and calculated deposition coefficients.

Table S2. Calculated filtration length needed for removing each $\mathrm{Hg}$ species to different targets.

Figure S1. Tracer test breakthrough curves.

Figure S2. Illustration of the method used to determine the deposition rate of the mobile phase from the column deposition profiles.

Figure S3. Fitting of mean $-\ln \left(\mathrm{C} / \mathrm{C}_{0}\right)$ vs. $\mathrm{L}$ for $\mathrm{Hg}$ species to determine $k_{d \text {,mobile. }}$

Figure S4. Dissolved $\mathrm{Hg}(\mathrm{II})$ species (denoted as $\left.\mathrm{Hg}(\mathrm{II})^{*}\right)$ under simulated rainwater $(0.005 \mathrm{M}$ $\mathrm{NaCl})$ condition.

Figure S5. Dissolved $\mathrm{Hg}$ (II) species (denoted as $\left.\mathrm{Hg}(\mathrm{II})^{*}\right)$ under simulated leachate $(0.2 \mathrm{M} \mathrm{NaCl}$, $147 \mathrm{mg} \mathrm{C} / \mathrm{L}$ ) condition.

Figure S6. Intensity-weighted $\mathrm{HgS}$ hydrodynamic radius determined from DLS. 
Figure S7. The change in measured hydrodynamic radius vs. time for $\mathrm{HgS}$ particles in two different column influent solutions.

Figure S8. Representative breakthrough curves and deposition profiles for the four different $\mathrm{Hg}$ species in the unsaturated sand column. Medium: \#50 Unimin sand.

Figure S9. Representative breakthrough curves and deposition profiles for the four different $\mathrm{Hg}$ species in the unsaturated sand column. Medium: \#50 Unimin sand (98 wt $\%)+$ clay (2 wt $\%)$.

Figure S10. Representative breakthrough curves and deposition profiles for the four different $\mathrm{Hg}$ species in the unsaturated sand column. Medium: low TOC soil from Alameda point, CA.

Figure S11. Representative breakthrough curves and deposition profiles for the four different $\mathrm{Hg}$ species in the unsaturated sand column. Medium: high TOC soil from Pittsburgh, PA. 
The hydraulic conductivity of the column medium was calculated using the equation S1,

$$
K=\frac{V L}{A t h}
$$

$K=$ Hydraulic conductivity at $20^{\circ} \mathrm{C}(\mathrm{cm} / \mathrm{s})$

$V=$ Volume of discharge $\left(\mathrm{cm}^{3}\right)$

$L=$ Length of column $(\mathrm{cm})$

$A=$ Cross section area $\left(\mathrm{cm}^{2}\right)$

$t=$ Time of discharge (s)

$h=$ Hydraulic head difference $(\mathrm{cm})$

Given the flow rate of $0.37 \mathrm{~mL} / \mathrm{min}$ and effective porosity determined by tracer test results (Figure S1), the average linear velocity of water was calculated using equation S2,

$$
v_{x}=\frac{Q}{n_{e} A}
$$

$v_{x}=$ Average linear velocity $(\mathrm{cm} / \mathrm{s})$

$Q=$ Flow rate $(\mathrm{mL} / \mathrm{min})$

$\mathrm{A}=$ Cross section area $\left(\mathrm{cm}^{2}\right)$

$n_{e}=$ Effective porosity

Given $L, v_{x}, \mathrm{C} / \mathrm{C}_{0}$ and $t$, the coefficient of hydrodynamic dispersion can be determined by least squares fitting of the tracer test breakthrough data using equation S3.

$$
C=\frac{C_{0}}{2}\left[\operatorname{erfc}\left(\frac{L-v_{x} t}{2 \sqrt{D_{L} t}}\right)+\exp \left(\frac{v_{x} L}{D_{L}}\right) \operatorname{erfc}\left(\frac{L+v_{x} t}{2 \sqrt{D_{L} t}}\right)\right]
$$

$C=$ Solute concentration at time $t$ 
$C_{0}=$ Initial solute concentration

$L=$ Transport distance $(\mathrm{cm})$

$D_{L}=$ Coefficient of hydrodynamic dispersion

erfc $=$ Complementary error function 


\section{S2. $\mathrm{Hg}(\mathrm{II}) *$ Speciation Analysis}

$\mathrm{Hg}(\mathrm{II}) *$ concentration was calculated by assuming the added $\mathrm{Hg}$ was well mixed in the top layer of the column (eqn S3).

$$
C_{H g(I I)}=\frac{M_{(H g(I) \text { doped })}}{V_{\text {pore volume in the top layer of the column }}}
$$

The Hg speciation was determined using Visual MINTEQ by assuming equilibrium conditions were reached. The HgDOM species was calculated by assuming the reaction of below: $\mathrm{Hg}(\mathrm{II})^{*}+\mathrm{DOM}=\mathrm{HgDOM} \quad \log \mathrm{K}=23.4 \mathrm{~L} / \mathrm{kg}^{[1]}$ 
Table S1. Column experiment parameters and calculated deposition rates.

\begin{tabular}{|c|c|c|c|c|c|}
\hline $\begin{array}{c}\text { Column } \\
\text { media }\end{array}$ & $\begin{array}{l}\text { Influent } \\
\text { solution }\end{array}$ & Hg species & $\frac{q}{N_{0}} \int_{0}^{t_{f}} C(t) d t^{a}$ & $\begin{array}{l}k_{\text {d,overall }} \\
\left(\mathrm{min}^{-1}\right)\end{array}$ & $\begin{array}{l}k_{d, m o b i l e} \\
\left(\text { min }^{-1}\right)\end{array}$ \\
\hline \multirow{8}{*}{ \#50sand } & \multirow{4}{*}{$5 \mathrm{mM} \mathrm{NaCl}$} & $\mathrm{Hg}(\mathrm{II})^{*}$ & $39 \%$ & 0.0207 & $\begin{array}{c}0.0165 \\
\pm 0.0014\end{array}$ \\
\hline & & $\mathrm{Hg}-\mathrm{DOM}$ & $25 \%$ & 0.0298 & $\begin{array}{l}0.0249 \\
\pm 0.003 \\
\end{array}$ \\
\hline & & $\mathrm{Hg}(0)$ & $26 \%$ & 0.0295 & $\begin{array}{c}0.0158 \\
\pm 0.0005\end{array}$ \\
\hline & & $\mathrm{HgS}$ & $0.4 \%$ & 0.1406 & $\begin{array}{c}0.0837 \\
\pm 0.0036 \\
\end{array}$ \\
\hline & \multirow{4}{*}{$\begin{array}{l}\text { 200mM NaCl, } \\
147 \mathrm{mg} \mathrm{C} / \mathrm{L} \\
\text { humic acid }\end{array}$} & $\mathrm{Hg}(\mathrm{II}) *$ & $96 \%$ & 0.0009 & $\begin{array}{c}0.0007 \\
\pm 0.0001\end{array}$ \\
\hline & & Hg-DOM & $88 \%$ & 0.0029 & $\begin{array}{c}0.0022 \\
\pm 0.0001\end{array}$ \\
\hline & & $\operatorname{Hg}(0)$ & $94 \%$ & 0.0014 & $\begin{array}{c}0.0003 \\
\pm 0\end{array}$ \\
\hline & & $\mathrm{HgS}$ & $12 \%$ & 0.0479 & $\begin{array}{c}0.0298 \\
\pm .002 \\
\end{array}$ \\
\hline \multirow{8}{*}{ \#50sand+clay } & \multirow{4}{*}{$5 \mathrm{mM} \mathrm{NaCl}$} & $\mathrm{Hg}(\mathrm{II})^{*}$ & $6 \%$ & 0.0611 & $\begin{array}{c}0.0377 \\
\pm 0.0083 \\
\end{array}$ \\
\hline & & $\mathrm{Hg}-\mathrm{DOM}$ & $14 \%$ & 0.0414 & $\begin{array}{c}0.0345 \\
\pm 0.0013\end{array}$ \\
\hline & & $\mathrm{Hg}(0)$ & $0.04 \%$ & 0.1630 & $\begin{array}{c}0.0825 \\
\pm 0.01\end{array}$ \\
\hline & & $\mathrm{HgS}$ & $0 \%$ & $\mathrm{~N} / \mathrm{A}$ & $\begin{array}{c}0.0925 \\
\pm 0.0119\end{array}$ \\
\hline & \multirow{4}{*}{$\begin{array}{l}\text { 200mM NaCl, } \\
147 \mathrm{mg} \mathrm{C} / \mathrm{L} \\
\text { humic acid }\end{array}$} & $\mathrm{Hg}(\mathrm{II})^{*}$ & $91 \%$ & 0.0021 & $\begin{array}{c}0.0023 \\
\pm 0.0001 \\
\end{array}$ \\
\hline & & $\mathrm{Hg}-\mathrm{DOM}$ & $81 \%$ & 0.0054 & $\begin{array}{l}0.0035 \\
\pm 0.001 \\
\end{array}$ \\
\hline & & $\operatorname{Hg}(0)$ & $22 \%$ & 0.0327 & $\begin{array}{c}0.018 \\
\pm 0.0013 \\
\end{array}$ \\
\hline & & $\mathrm{HgS}$ & $5 \%$ & 0.0656 & $\begin{array}{c}0.0395 \\
\pm 0.0027 \\
\end{array}$ \\
\hline \multirow{8}{*}{ Low TOC soil } & \multirow{4}{*}{$5 \mathrm{mM} \mathrm{NaCl}$} & $\mathrm{Hg}(\mathrm{II})^{*}$ & $2 \%$ & 0.0826 & $\begin{array}{c}0.0742 \\
\pm 0.0037\end{array}$ \\
\hline & & Hg-DOM & $69 \%$ & 0.0078 & $\begin{array}{c}0.0056 \\
\pm 0.0001 \\
\end{array}$ \\
\hline & & $\mathrm{Hg}(0)$ & $42 \%$ & 0.0183 & $\begin{array}{l}0.0133 \\
\pm 0.005\end{array}$ \\
\hline & & $\mathrm{HgS}$ & $5 \%$ & 0.0632 & $\begin{array}{c}0.0335 \\
\pm 0.0034\end{array}$ \\
\hline & \multirow{4}{*}{$\begin{array}{c}\text { 200mM NaCl, } \\
147 \mathrm{mg} \mathrm{C} / \mathrm{L} \\
\text { humic acid }\end{array}$} & $\mathrm{Hg}(\mathrm{II})^{*}$ & $45 \%$ & 0.0169 & $\begin{array}{c}0.0111 \\
\pm 0.0008\end{array}$ \\
\hline & & $\mathrm{Hg}-\mathrm{DOM}$ & $78 \%$ & 0.0052 & $\begin{array}{c}0.0035 \\
\pm 0.0001 \\
\end{array}$ \\
\hline & & $\mathrm{Hg}(0)$ & $52 \%$ & 0.0138 & $\begin{array}{c}0.0088 \\
\pm 0.0001 \\
\end{array}$ \\
\hline & & $\mathrm{HgS}$ & $11 \%$ & 0.0466 & $\begin{array}{c}0.0279 \\
\pm 0.0007\end{array}$ \\
\hline High TOC soil & $5 \mathrm{mM} \mathrm{NaCl}$ & $\mathrm{Hg}(\mathrm{II})^{*}$ & $0 \%$ & $\mathrm{~N} / \mathrm{A}$ & $\begin{array}{r}0.0652 \\
\pm .0652\end{array}$ \\
\hline
\end{tabular}


Table S1 (continued). Column experiment parameters and calculated deposition rates.

\begin{tabular}{|c|c|c|c|c|c|}
\hline $\begin{array}{c}\text { Column } \\
\text { media }\end{array}$ & $\begin{array}{l}\text { Influent } \\
\text { solution }\end{array}$ & Hg species & $\frac{q}{N_{0}} \int_{0}^{t_{f}} C(t) d t^{a}$ & $\begin{array}{l}k_{d, o v e r a l l} \\
\left(\mathrm{~min}^{-1}\right)\end{array}$ & $\begin{array}{l}k_{d, m o b i l e} \\
\left(\mathrm{~min}^{-1}\right)\end{array}$ \\
\hline & & $\mathrm{Hg}-\mathrm{DOM}$ & $5 \%$ & 0.0632 & $\begin{array}{r}0.0382 \\
\pm .0382 \\
\end{array}$ \\
\hline & & $\operatorname{Hg}(0)$ & $0 \%$ & N/A & $\begin{array}{r}0.0575 \\
\pm .0575 \\
\end{array}$ \\
\hline & & $\mathrm{HgS}$ & $0 \%$ & N/A & $\begin{array}{r}0.0526 \\
\pm .0526\end{array}$ \\
\hline & \multirow{4}{*}{$\begin{array}{c}200 \mathrm{mM} \mathrm{NaCl} \text {, } \\
147 \mathrm{mg} \mathrm{C} / \mathrm{L} \\
\text { humic acid }\end{array}$} & $\mathrm{Hg}(\mathrm{II})^{*}$ & $13 \%$ & 0.0431 & $\begin{array}{c}0.0357 \\
\pm 0.0031 \\
\end{array}$ \\
\hline & & $\mathrm{Hg}-\mathrm{DOM}$ & $20 \%$ & 0.0340 & $\begin{array}{c}0.0261 \\
\pm 0.0027 \\
\end{array}$ \\
\hline & & $\mathrm{Hg}(0)$ & $11 \%$ & 0.0466 & $\begin{array}{r}0.0296 \\
\pm 0.0032 \\
\end{array}$ \\
\hline & & $\mathrm{HgS}$ & $2 \%$ & 0.0826 & $\begin{array}{l}0.0431 \\
\pm 0.067\end{array}$ \\
\hline
\end{tabular}


Table S2. Calculated filtration length needed for removing each $\mathrm{Hg}$ species to different targets.

\begin{tabular}{|c|c|c|c|c|c|c|}
\hline \multirow{2}{*}{$\begin{array}{l}\text { Column } \\
\text { media }\end{array}$} & \multirow{2}{*}{$\begin{array}{l}\text { Influent } \\
\text { solution }\end{array}$} & \multirow[b]{2}{*}{ Hg species } & \multicolumn{4}{|c|}{ Filter length needed (m) } \\
\hline & & & $\begin{array}{l}99 \% \\
\text { removal }\end{array}$ & $\begin{array}{l}99.9 \% \\
\text { removal }\end{array}$ & $\begin{array}{l}99.99 \% \\
\text { removal }\end{array}$ & $\begin{array}{l}99.999 \% \\
\text { removal }\end{array}$ \\
\hline \multirow{8}{*}{ \#50sand } & \multirow{4}{*}{$5 \mathrm{mM} \mathrm{NaCl}$} & $\mathrm{Hg}(\mathrm{II})^{*}$ & $0.55 \pm 0.04$ & $0.83 \pm 0.07$ & $1.11 \pm 0.09$ & $1.38 \pm 0.11$ \\
\hline & & Hg-DOM & $0.36 \pm 0$ & $0.53 \pm 0.01$ & $0.71 \pm 0.01$ & $0.89 \pm 0.01$ \\
\hline & & $\mathrm{Hg}(0)$ & $0.57 \pm 0.02$ & $0.86 \pm 0.07$ & $1.15 \pm 0.04$ & $1.43 \pm 0.05$ \\
\hline & & $\mathrm{HgS}$ & $0.13 \pm 0.01$ & $0.19 \pm 0.01$ & $0.25 \pm 0.01$ & $0.32 \pm 0.01$ \\
\hline & \multirow{4}{*}{$\begin{array}{c}200 \mathrm{mM} \mathrm{NaCl} \\
147 \mathrm{mg} \mathrm{C} / \mathrm{L} \\
\text { humic acid }\end{array}$} & $\mathrm{Hg}(\mathrm{II})^{*}$ & $12.81 \pm 1.2$ & $19.22 \pm 1.81$ & $25.63 \pm 2.41$ & $32.03 \pm 3.01$ \\
\hline & & Hg-DOM & $4.18 \pm 0.24$ & $6.27 \pm 0.35$ & $8.36 \pm 0.47$ & $10.44 \pm 0.59$ \\
\hline & & $\mathrm{Hg}(0)$ & $28.26 \pm 0.74$ & $42.39 \pm 1.1$ & $56.52 \pm 1.47$ & $70.66 \pm 1.84$ \\
\hline & & $\mathrm{HgS}$ & $0.32 \pm 0.02$ & $0.47 \pm 0.03$ & $0.63 \pm 0.04$ & $0.79 \pm 0.05$ \\
\hline \multirow{8}{*}{$\# 50$ sand+clay } & \multirow{4}{*}{$5 \mathrm{mM} \mathrm{NaCl}$} & $\mathrm{Hg}(\mathrm{II})^{*}$ & $0.24 \pm 0.05$ & $0.37 \pm 0.08$ & $0.49 \pm 0.11$ & $0.61 \pm 0.14$ \\
\hline & & $\mathrm{Hg}$-DOM & $0.25 \pm 0.01$ & $0.38 \pm 0.01$ & $0.51 \pm 0.02$ & $0.63 \pm 0.02$ \\
\hline & & $\mathrm{Hg}(0)$ & $0.1 \pm 0.01$ & $0.16 \pm 0.02$ & $0.21 \pm 0.03$ & $0.26 \pm 0.03$ \\
\hline & & $\mathrm{HgS}$ & $0.1 \pm 0.01$ & $0.15 \pm 0.02$ & $0.2 \pm 0.03$ & $0.25 \pm 0.03$ \\
\hline & \multirow{4}{*}{$\begin{array}{c}200 \mathrm{mM} \mathrm{NaCl}, \\
147 \mathrm{mg} \mathrm{C} / \mathrm{L} \\
\text { humic acid }\end{array}$} & $\mathrm{Hg}(\mathrm{II})^{*}$ & $3.94 \pm 0.24$ & $5.91 \pm 0.36$ & $7.88 \pm 0.49$ & $9.85 \pm 0.61$ \\
\hline & & $\mathrm{Hg}$-DOM & $3.02 \pm 0.1$ & $4.53 \pm 0.15$ & $6.04 \pm 0.2$ & $7.55 \pm 0.25$ \\
\hline & & $\mathrm{Hg}(0)$ & $0.5 \pm 0.04$ & $0.75 \pm 0.05$ & $1 \pm 0.07$ & $1.25 \pm 0.09$ \\
\hline & & $\mathrm{HgS}$ & $0.23 \pm 0.02$ & $0.35 \pm 0.02$ & $0.46 \pm 0.03$ & $0.58 \pm 0.04$ \\
\hline \multirow{8}{*}{ Low TOC soil } & \multirow{4}{*}{$5 \mathrm{mM} \mathrm{NaCl}$} & $\mathrm{Hg}(\mathrm{II})^{*}$ & $0.12 \pm 0.01$ & $0.18 \pm 0.01$ & $0.24 \pm 0.01$ & $0.3 \pm 0.01$ \\
\hline & & Hg-DOM & $1.56 \pm 0.03$ & $2.35 \pm 0.05$ & $3.13 \pm 0.06$ & $3.91 \pm 0.08$ \\
\hline & & $\mathrm{Hg}(0)$ & $0.96 \pm 0.04$ & $1.44 \pm 0.06$ & $1.92 \pm 0.08$ & $2.4 \pm 0.1$ \\
\hline & & $\mathrm{HgS}$ & $0.15 \pm 0.01$ & $0.22 \pm 0.01$ & $0.3 \pm 0.02$ & $0.37 \pm 0.02$ \\
\hline & \multirow{4}{*}{$\begin{array}{c}200 \mathrm{mM} \mathrm{NaCl}, \\
147 \mathrm{mg} \mathrm{C} / \mathrm{L} \\
\text { humic acid }\end{array}$} & $\mathrm{Hg}(\mathrm{II})^{*}$ & $0.79 \pm 0.06$ & $1.18 \pm 0.08$ & $1.58 \pm 0.11$ & $1.97 \pm 0.14$ \\
\hline & & Hg-DOM & $2.51 \pm 0.06$ & $3.78 \pm 0.1$ & $5.03 \pm 0.13$ & $6.29 \pm 0.16$ \\
\hline & & $\mathrm{Hg}(0)$ & $0.99 \pm 0.01$ & $1.48 \pm 0.02$ & $1.98 \pm 0.02$ & $2.47 \pm 0.03$ \\
\hline & & $\mathrm{HgS}$ & $0.31 \pm 0.01$ & $0.47 \pm 0.01$ & $0.63 \pm 0.01$ & $0.78 \pm 0.02$ \\
\hline \multirow{8}{*}{ High TOC soil } & \multirow{4}{*}{$5 \mathrm{mM} \mathrm{NaCl}$} & $\mathrm{Hg}(\mathrm{II})^{*}$ & $0.14 \pm 0.03$ & $0.21 \pm 0.04$ & $0.27 \pm 0.05$ & $0.34 \pm 0.07$ \\
\hline & & $\mathrm{Hg}$-DOM & $0.23 \pm 0.05$ & $0.35 \pm 0.08$ & $0.47 \pm 0.1$ & $0.59 \pm 0.13$ \\
\hline & & $\mathrm{Hg}(0)$ & $0.15 \pm 0.02$ & $0.23 \pm 0.03$ & $0.31 \pm 0.4$ & $0.38 \pm 0.05$ \\
\hline & & $\mathrm{HgS}$ & $0.17 \pm 0.04$ & $0.26 \pm 0.07$ & $0.34 \pm 0.09$ & $0.43 \pm 0.11$ \\
\hline & \multirow{4}{*}{$\begin{array}{c}200 \mathrm{mM} \mathrm{NaCl} \\
147 \mathrm{mg} \mathrm{C} / \mathrm{L} \\
\text { humic acid }\end{array}$} & $\mathrm{Hg}(\mathrm{II})^{*}$ & $0.25 \pm 0.02$ & $0.38 \pm 0.03$ & $0.51 \pm 0.04$ & $0.63 \pm 0.05$ \\
\hline & & Hg-DOM & $0.34 \pm 0.03$ & $0.5 \pm 0.05$ & $0.67 \pm 0.07$ & $0.84 \pm 0.09$ \\
\hline & & $\mathrm{Hg}(0)$ & $0.29 \pm 0.03$ & $0.44 \pm 0.05$ & $0.58 \pm 0.06$ & $0.73 \pm 0.08$ \\
\hline & & $\mathrm{HgS}$ & $0.21 \pm 0.03$ & $0.32 \pm 0.05$ & $0.42 \pm 0.07$ & $0.53 \pm 0.08$ \\
\hline
\end{tabular}




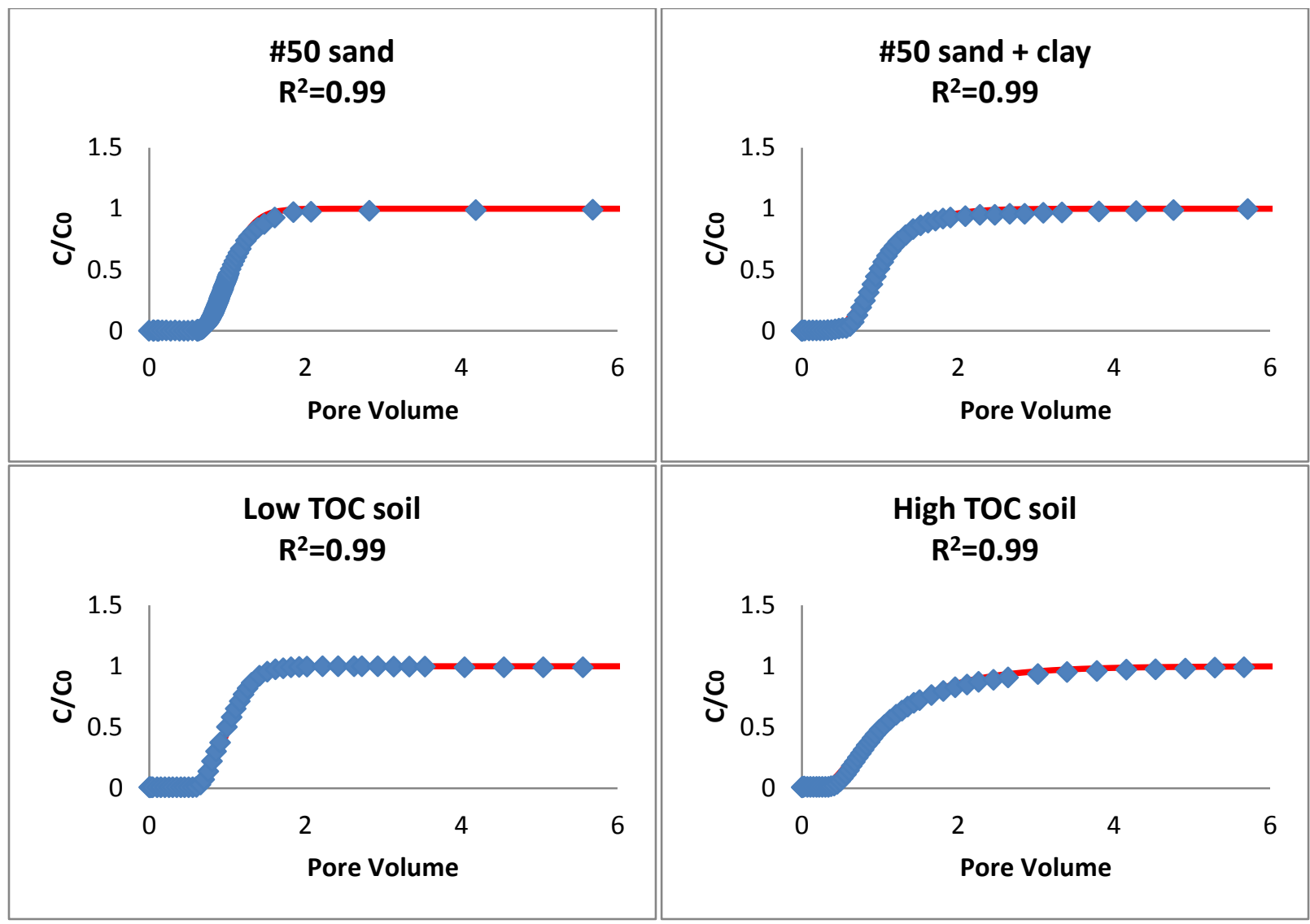

Figure S1. Tracer test breakthrough curves. Tracer: $20 \mathrm{mM} \mathrm{NaCl}$; Blue square symbols are the raw data collected by in-line conductivity detector and the red lines represent the least square fitting of the raw data using eqn S2. The similarity of the breakthrough curve shapes suggest a similar water distribution in the different soils. 


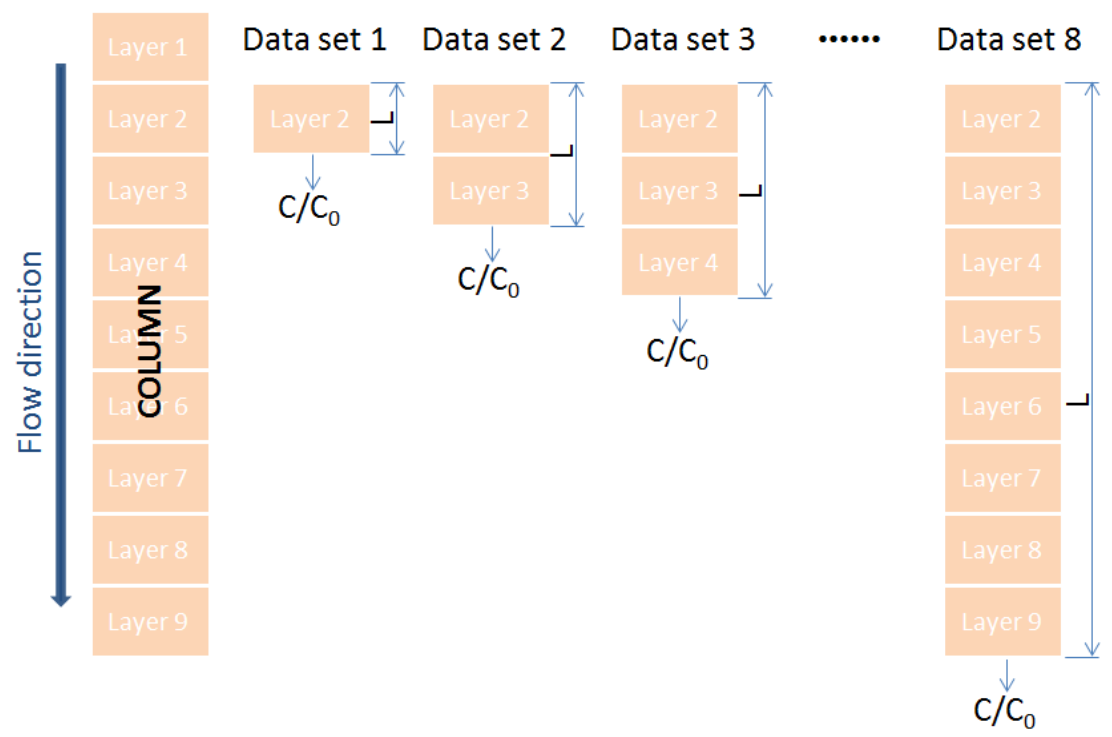

Figure S2. Illustration of the method used to determine the deposition rate coefficient for the mobile $\mathrm{Hg}$ mass introduced into Layer 1 for the column transport experiments. $C_{0}$ was the mobile $\mathrm{Hg}$ concentration determined from the difference in mass of $\mathrm{Hg}$ in Layer 1 at the end of the experiment compared to what was initially emplaced in this layer. $C$ was the mobile $\mathrm{Hg}$ concentration at the bottom of a layer $\mathrm{n}(\mathrm{n}=2-9)$ through a segment length of L. $C$ at the exit of each segment was calculated from a mass balance on that segment and all segments above it, i.e. was determined by subtracting the $\mathrm{Hg}$ mass deposited in $\mathrm{n}$ segments from total $\mathrm{Hg}$ mass entering layer 2. For all $\mathrm{Hg}$ species under a specific column condition, a plot of $\ln \left(C / C_{0}\right)$ vs. $L$ was made, and the slope of the line formed was used to determine the mobile deposition rate according to equation 2 . 


$$
\mathrm{Hg}(\mathrm{II})^{*} \quad \mathrm{Hg}-\mathrm{DOM} \quad \mathrm{Hg}(0) \quad \mathrm{HgS}
$$

\#50 sand, $5 \mathrm{mM} \mathrm{NaCl}$

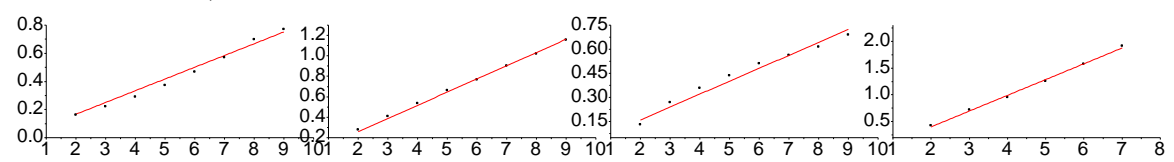

\#50 sand, $200 \mathrm{mM} \mathrm{NaCl}+1 \mathrm{mg} \mathrm{C} / \mathrm{L} \mathrm{DOM}$

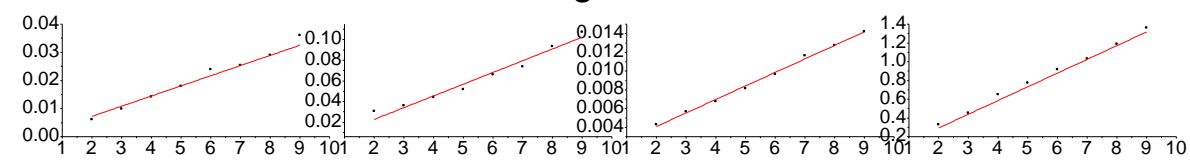

\#50 sand+clay, $5 \mathrm{mM} \mathrm{NaCl}$

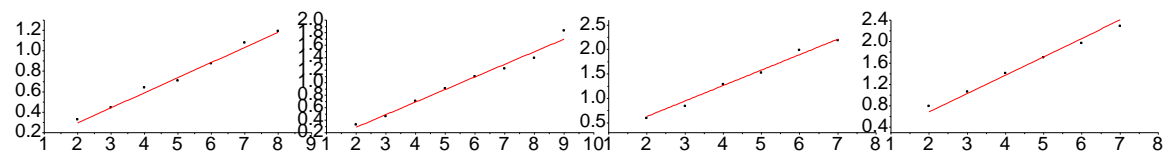

\#50 sand+clay, $200 \mathrm{mM} \mathrm{NaCl}+1 \mathrm{mg} \mathrm{C/L} \mathrm{DOM}$

$\frac{0}{0}$
$\frac{1}{1}$
$\frac{1}{0}$
$\frac{0}{\Sigma}$

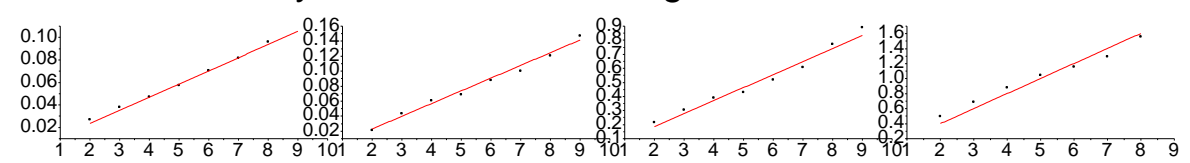

Low TOC soil, $5 \mathrm{mM} \mathrm{NaCl}$

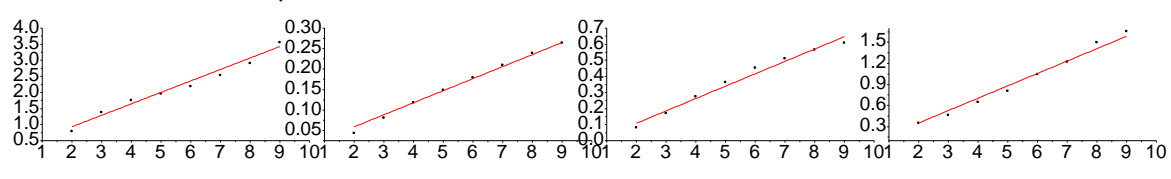

Low TOC soil, $200 \mathrm{mM} \mathrm{NaCl}+1 \mathrm{mg} \mathrm{C/L} \mathrm{DOM}$

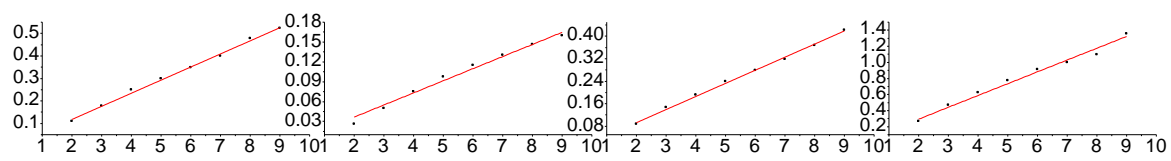

High TOC soil, $5 \mathrm{mM} \mathrm{NaCl}$

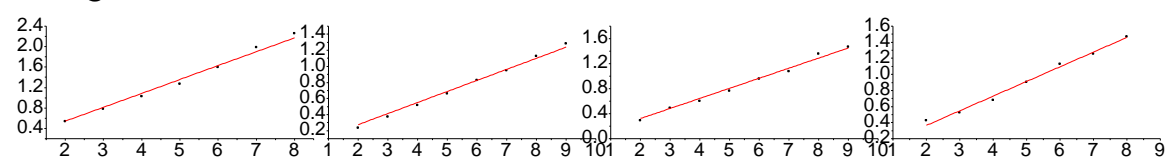

High TOC soil, $200 \mathrm{mM} \mathrm{NaCl}+1 \mathrm{mg} \mathrm{C/L} \mathrm{DOM}$

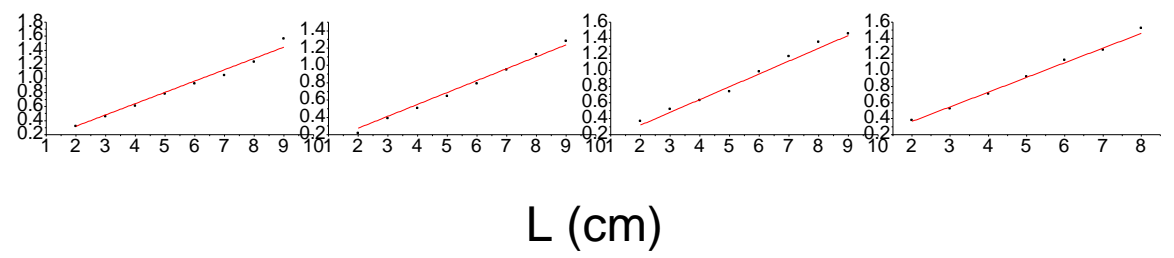

Figure S3. Fitting of mean $-\ln \left(\mathrm{C} / \mathrm{C}_{0}\right)$ vs. $\mathrm{L}$ for $\mathrm{Hg}$ species to determine $k_{\text {d,mobile }}$. 


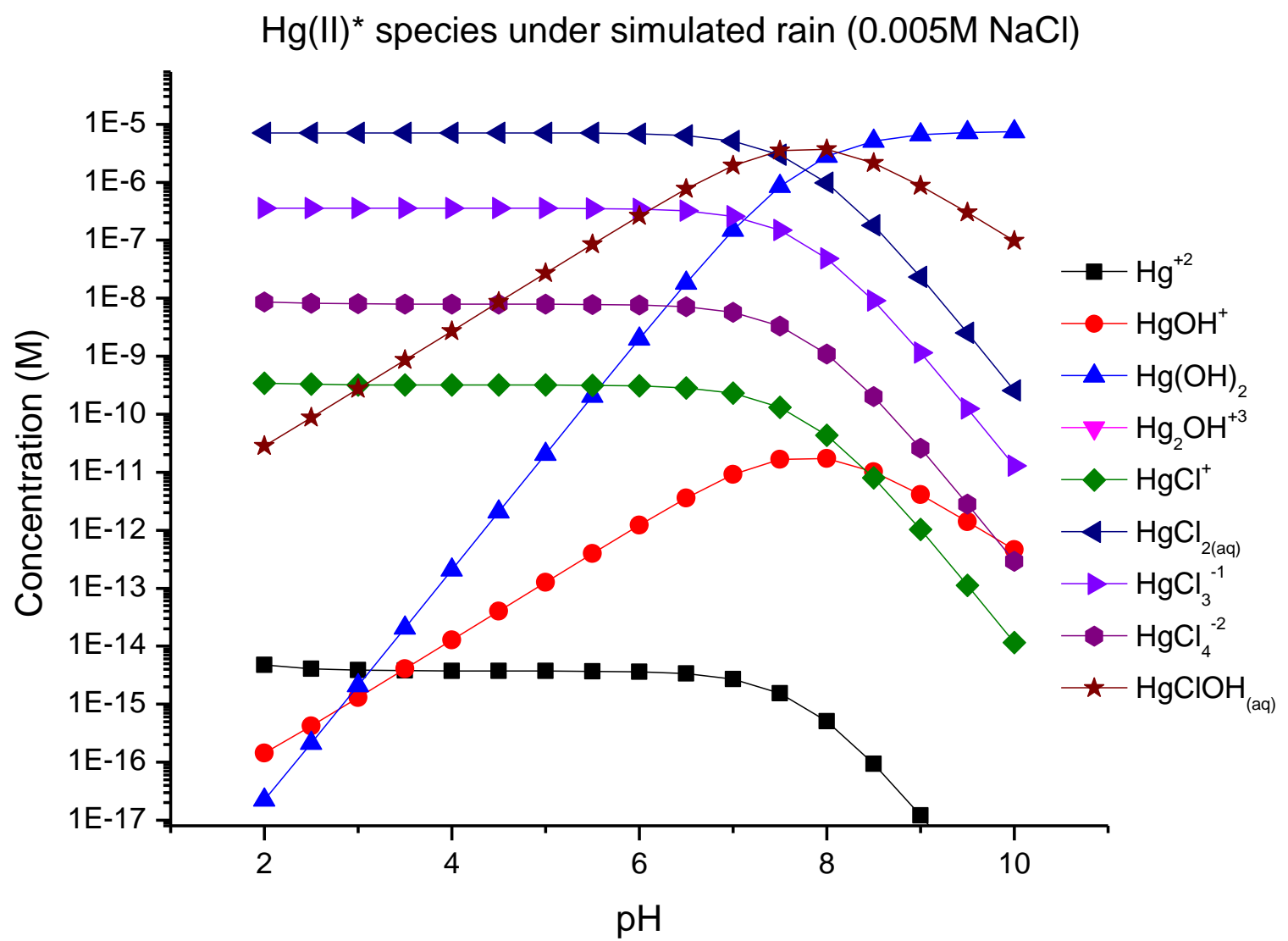

Figure S4. Dissolved $\mathrm{Hg}$ (II) species (denoted as $\left.\mathrm{Hg}(\mathrm{II})^{*}\right)$ under simulated rainwater $(0.005 \mathrm{M}$ $\mathrm{NaCl}$ ) condition. 
$\mathrm{Hg}(\mathrm{II})^{*}$ species under simulated leachate $(0.2 \mathrm{M} \mathrm{NaCl} 147 \mathrm{mg} \mathrm{C} / \mathrm{L})$

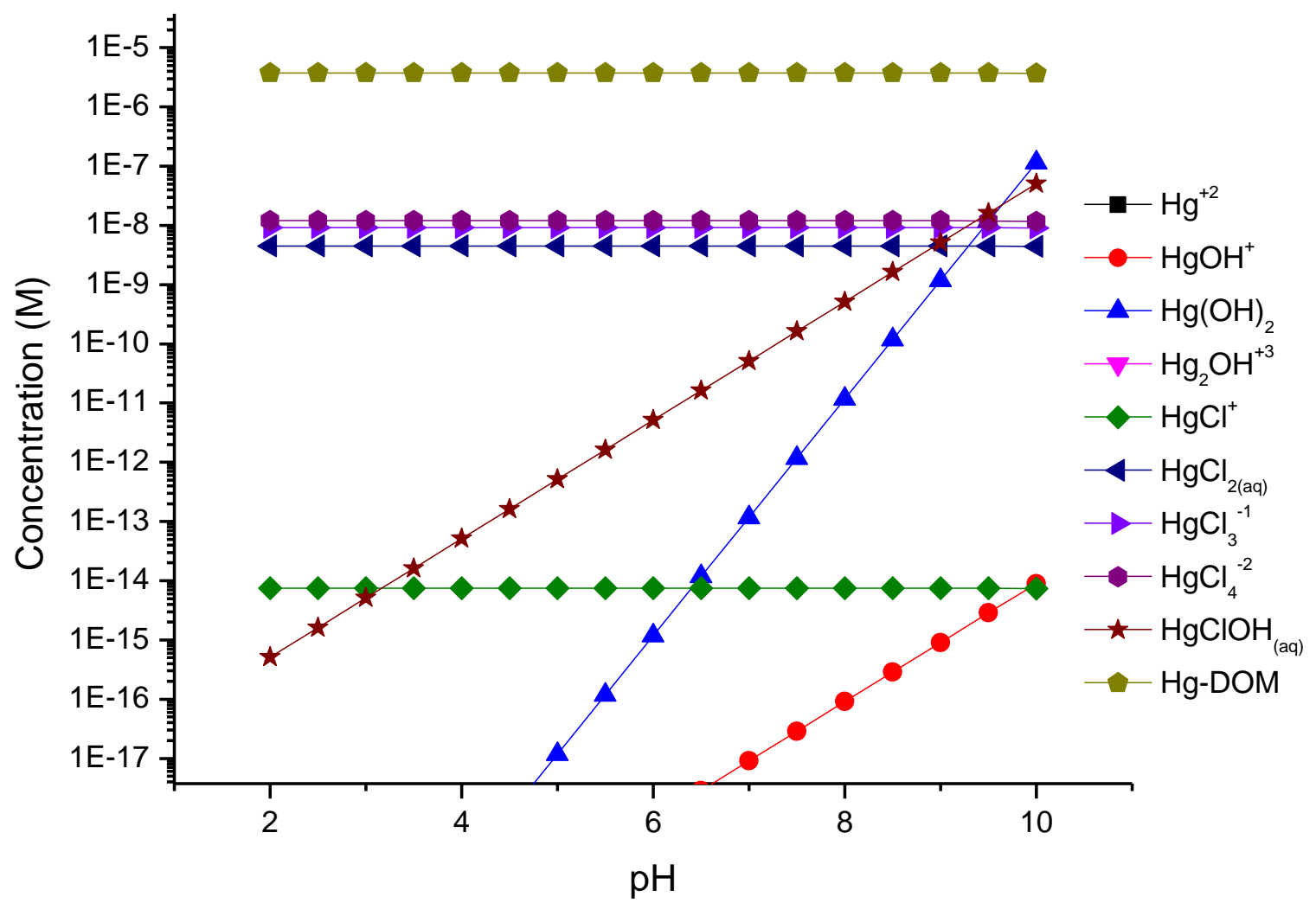

Figure S5. Dissolved $\mathrm{Hg}(\mathrm{II})$ species (denoted as $\left.\mathrm{Hg}(\mathrm{II})^{*}\right)$ under simulated leachate $(0.2 \mathrm{M} \mathrm{NaCl}$, $147 \mathrm{mg} \mathrm{C} / \mathrm{L}$ ) condition. 


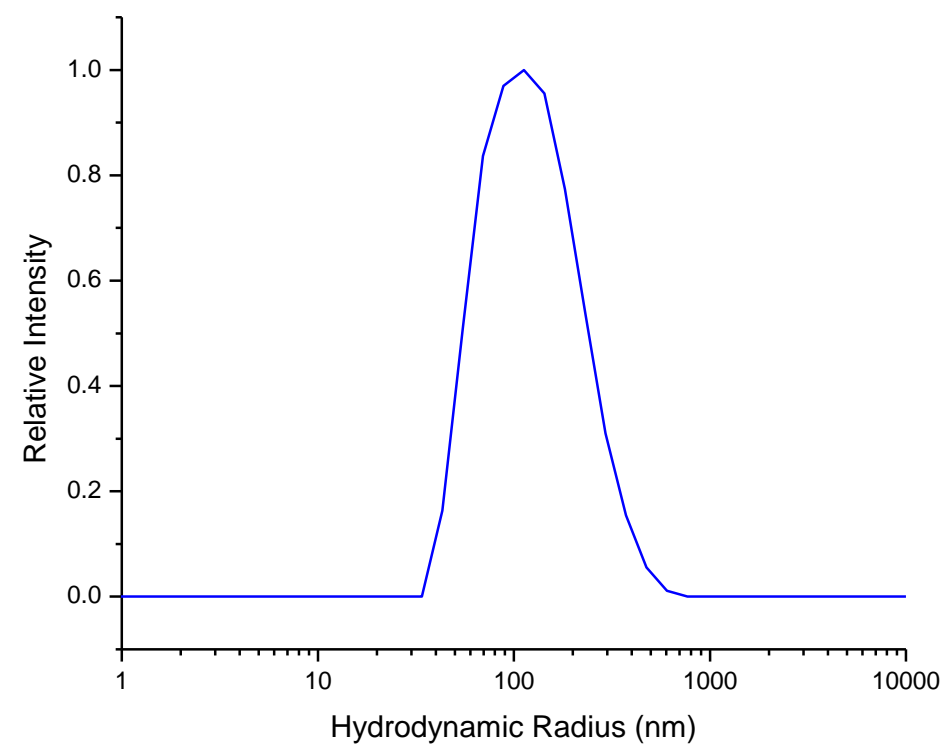

Figure S6. Intensity-weighted hydrodynamic radius of $\mathrm{HgS}$ nanoparticle stock solution as determined from DLS (polydispersity index $=0.35$ ). 


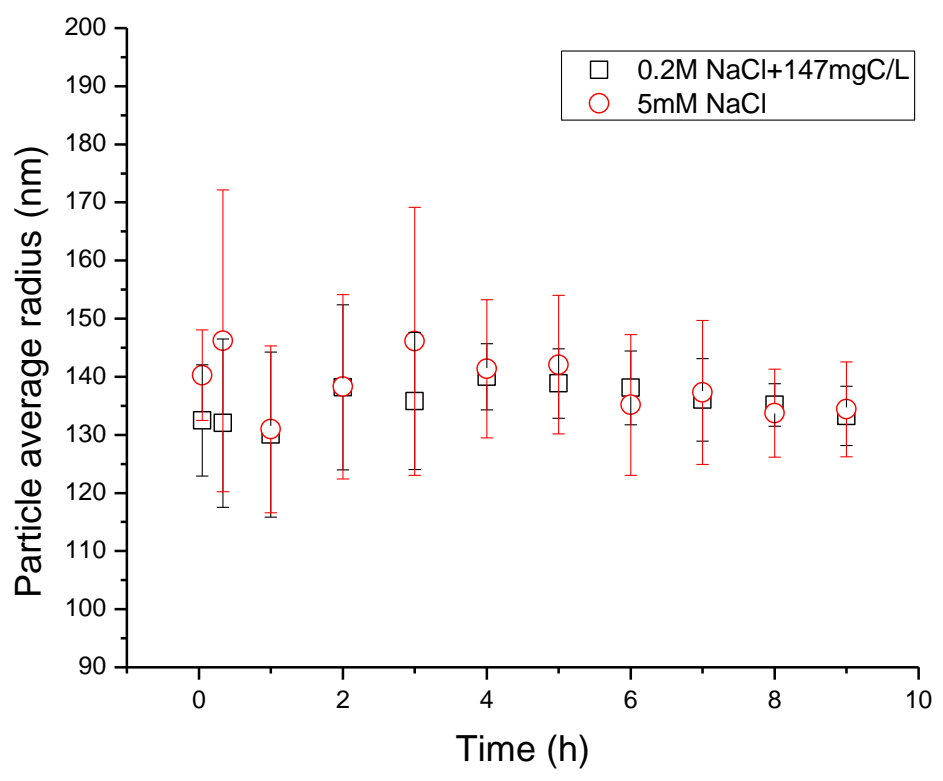

Figure S7. The change in measured hydrodynamic radius vs. time for $\mathrm{HgS}$ particles in two different column influent solutions, 1) $5 \mathrm{mM} \mathrm{NaCl}$ and 2) $200 \mathrm{mM} \mathrm{NaCl}+147 \mathrm{mg}$ carbon per L DOC. 

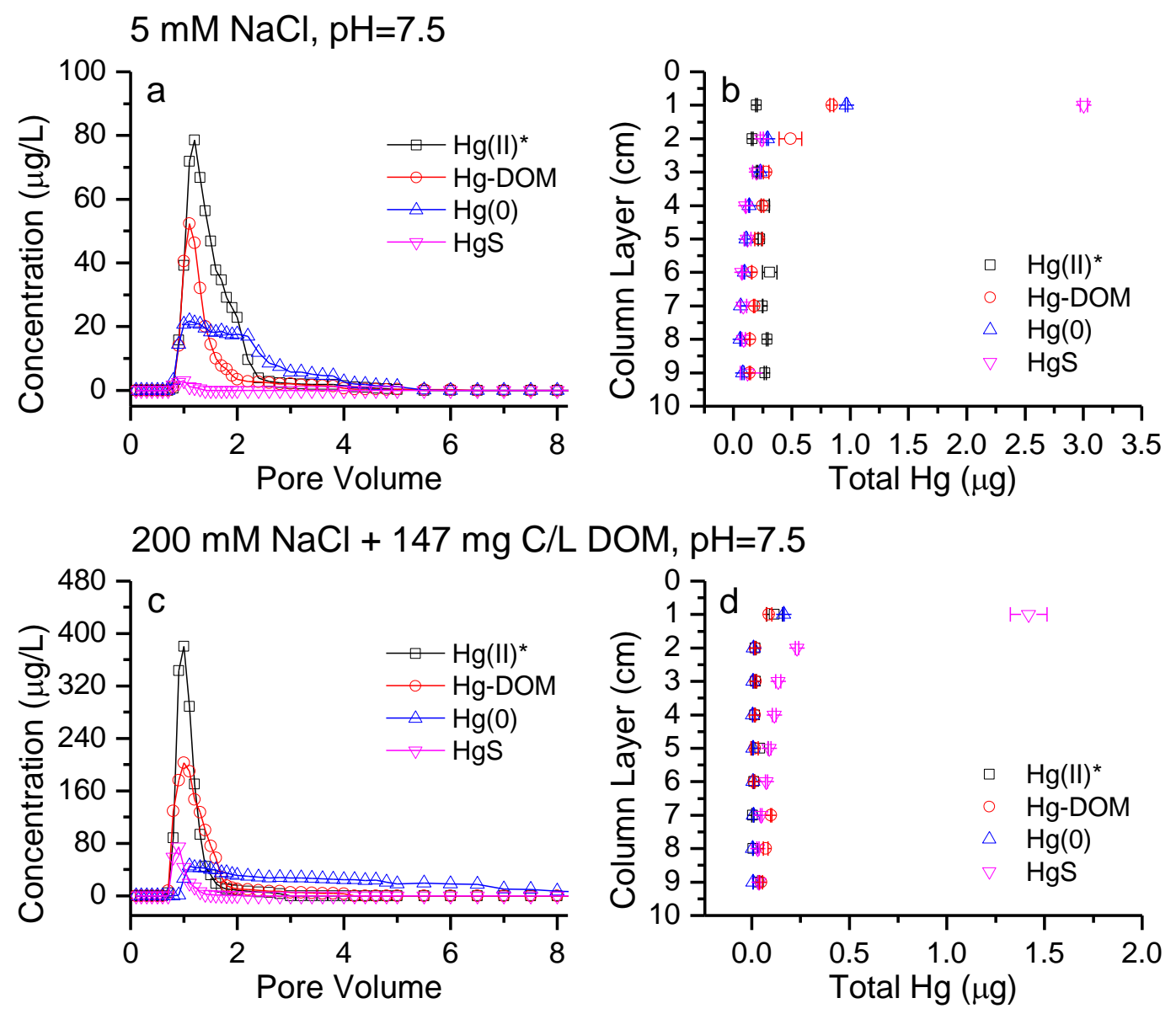

Figure S8. Representative breakthrough curves (a and c) and deposition profiles (b and d) for the four different $\mathrm{Hg}$ species in the unsaturated sand column. Column size: $9 \mathrm{~cm} \times$ 2.5cm; Medium: \#50 Unimin sand; Influent chemistry for a and b: $0.005 \mathrm{M} \mathrm{NaCl}$, pH 7.5; Influent chemistry for c and d: $200 \mathrm{mM} \mathrm{NaCl}+147 \mathrm{mg} \mathrm{C} / \mathrm{L} \mathrm{DOC}, \mathrm{pH}$ 7.5. Lines are not model fits of data. They are only meant to guide the eye. 

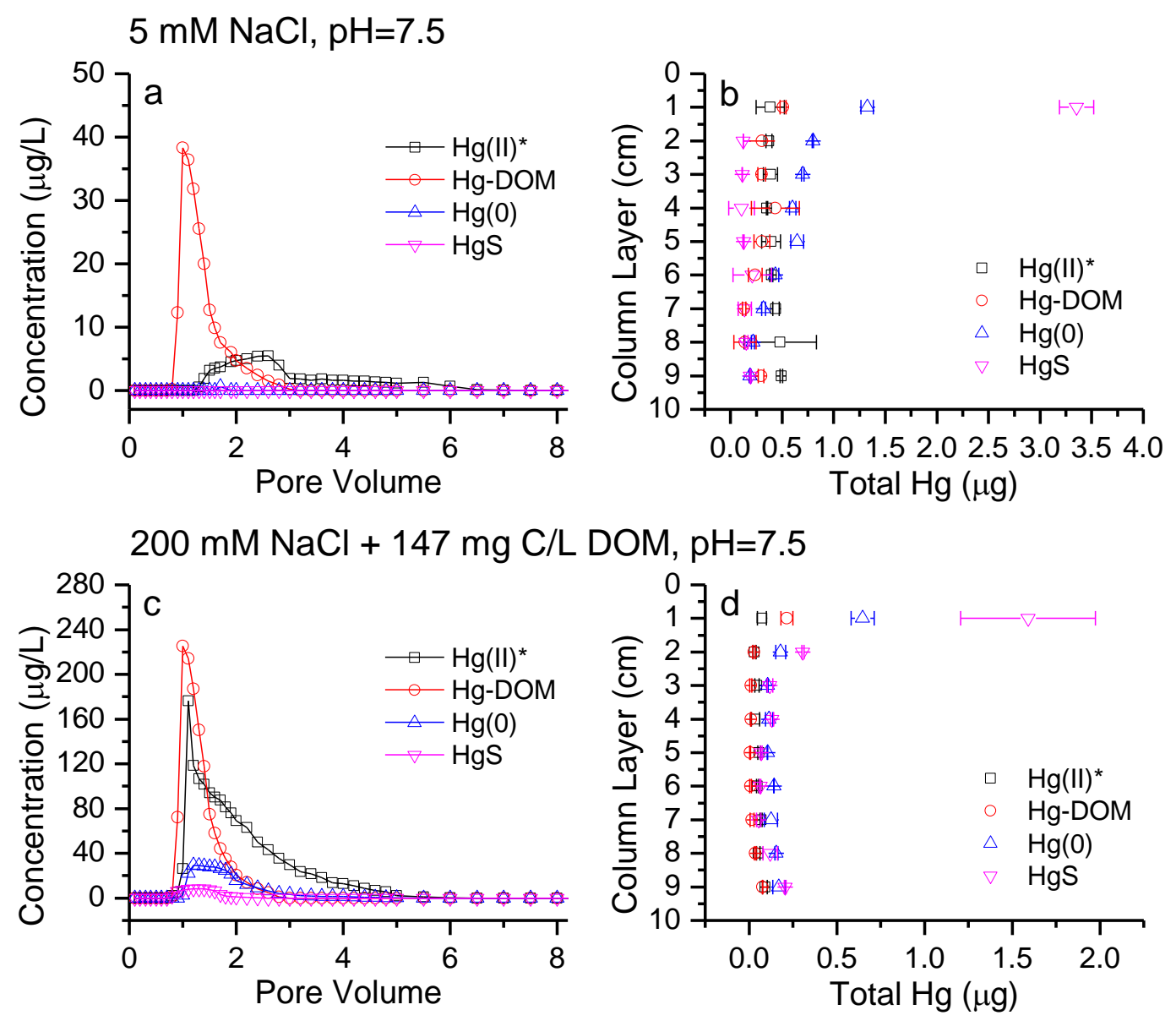

Figure S9. Representative breakthrough curves (a and c) and deposition profiles (b and d) for the four different $\mathrm{Hg}$ species in the unsaturated sand column. Column size: $9 \mathrm{~cm} \times$ 2.5cm; Medium: \#50 Unimin sand (98 wt\%) + clay (2 wt $\%)$; Influent chemistry for a and b: $0.005 \mathrm{M} \mathrm{NaCl}, \mathrm{pH} 7.5$; Influent chemistry for c and d: $200 \mathrm{mM} \mathrm{NaCl}+147$ mg C/L DOC, $\mathrm{pH}$ 7.5. Lines are not model fits of data. They are only meant to guide the eye. 

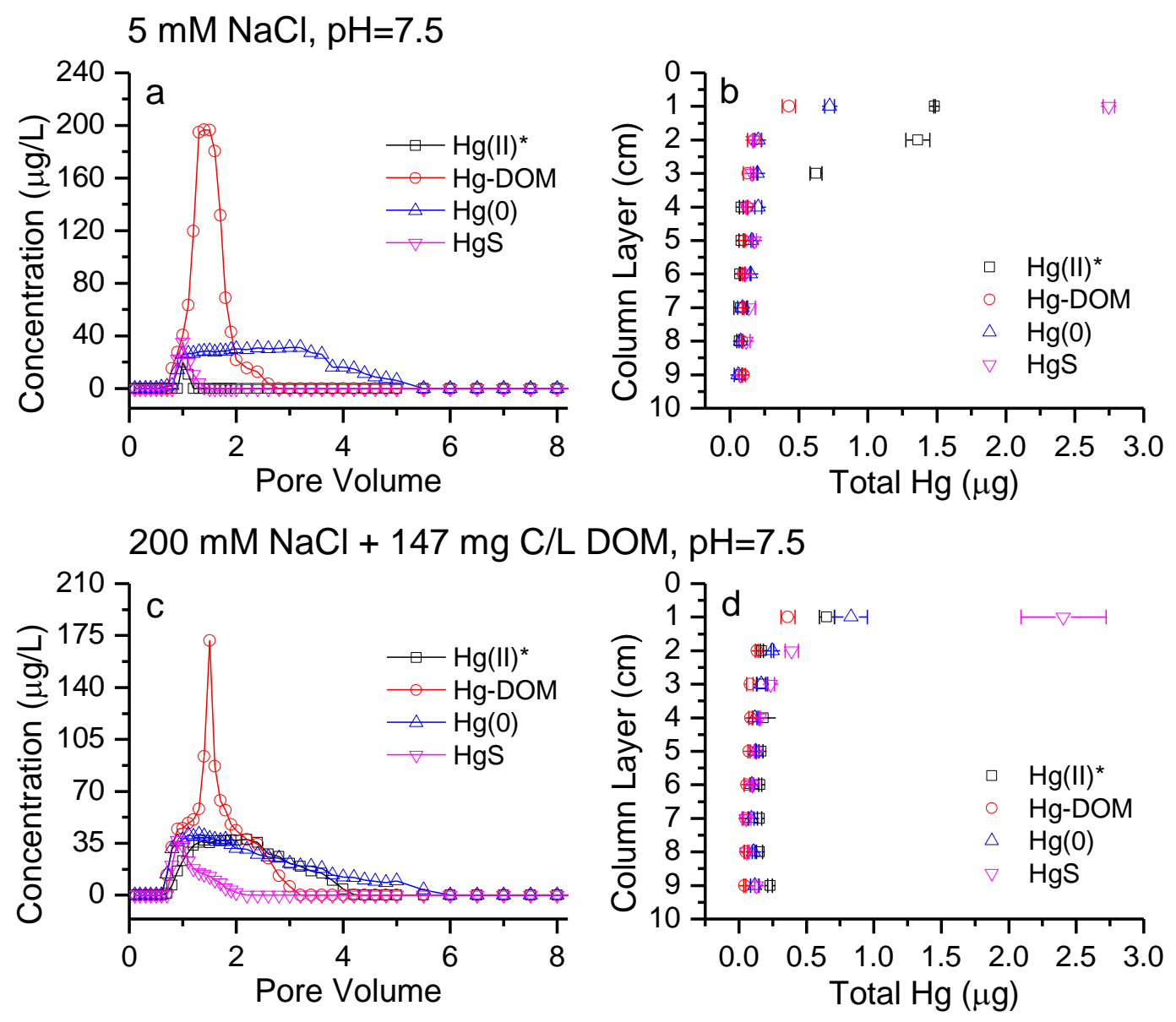

Figure S10. Representative breakthrough curves (a and c) and deposition profiles ( $b$ and d) for the four different $\mathrm{Hg}$ species in the unsaturated sand column. Column size: $9 \mathrm{~cm} \times$ 2.5cm; Medium: low TOC soil from Alameda point, CA; Influent chemistry for a and b: $0.005 \mathrm{M} \mathrm{NaCl}, \mathrm{pH} 7.5$; Influent chemistry for c and d: $200 \mathrm{mM} \mathrm{NaCl}+147$ $\mathrm{mg} \mathrm{C} / \mathrm{L}$ DOC, $\mathrm{pH}$ 7.5. Lines are not model fits of data. They are only meant to guide the eye. 

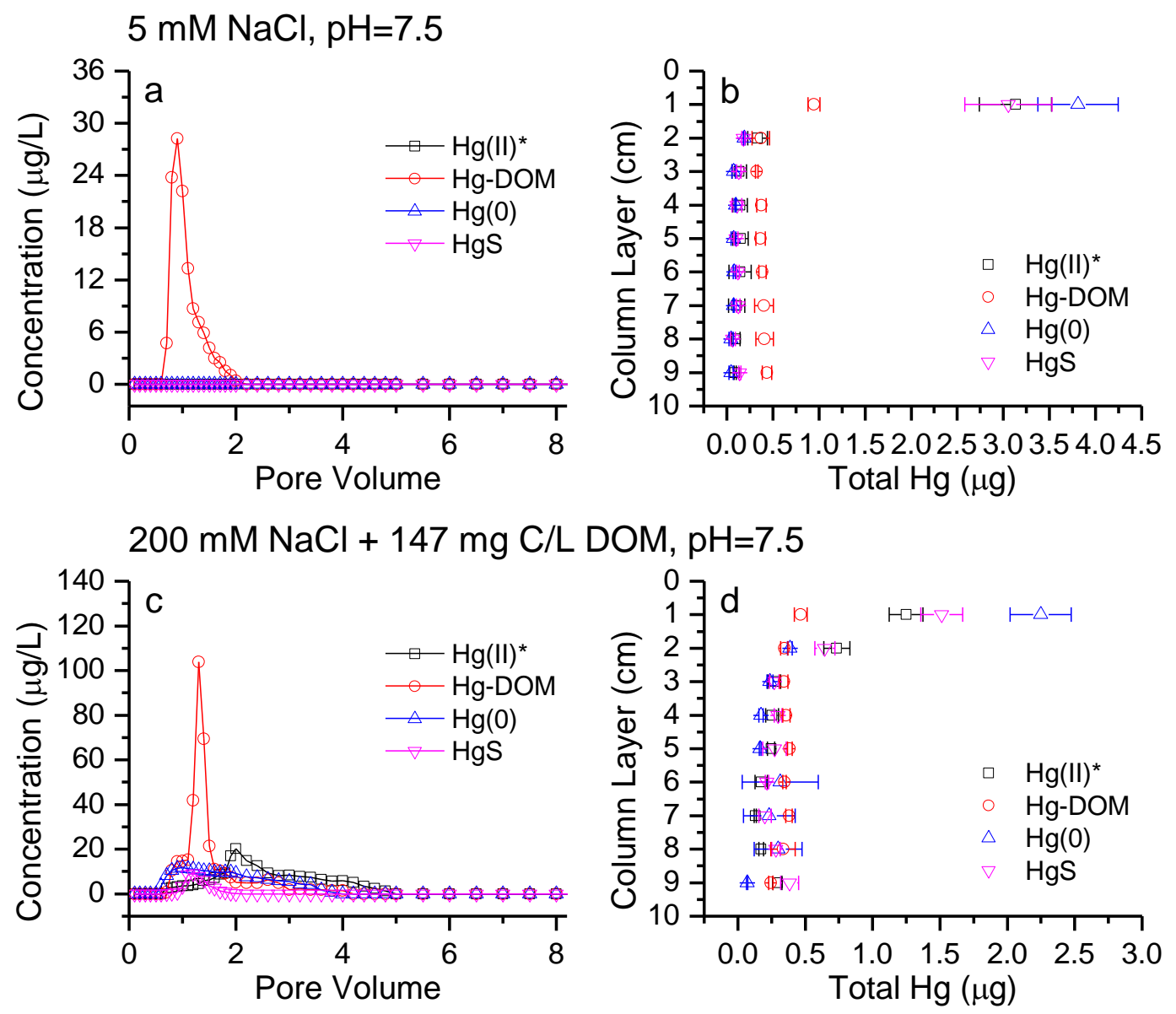

Figure S11. Representative breakthrough curves (a and c) and deposition profiles ( $b$ and d) for the four different $\mathrm{Hg}$ species in the unsaturated sand column. Column size: $9 \mathrm{~cm} \times$ 2.5cm; Medium: high TOC soil from Pittsburgh, PA; Influent chemistry for a and b: $0.005 \mathrm{M} \mathrm{NaCl}, \mathrm{pH}$ 7.5; Influent chemistry for c and d: $200 \mathrm{mM} \mathrm{NaCl}+147 \mathrm{mg} \mathrm{C} / \mathrm{L}$ DOC, $\mathrm{pH}$ 7.5. Lines are not model fits of data. They are only meant to guide the eye. 


\section{Reference}

1. Haitzer, M., G.R. Aiken, and J.N. Ryan, Binding of mercury (II) to dissolved organic matter: the role of the mercury-to-DOM concentration ratio. Environmental science \& technology, 2002. 36(16): p. 3564-3570. 